\title{
L'involontaire psychique du Projet Professionnel Personnalisé
}

Jean-Luc Vannier

\section{(2) OpenEdition}

1 Journals

Édition électronique

URL : http://journals.openedition.org/communicationorganisation/1491

DOI : 10.4000/communicationorganisation. 1491

ISSN : $1775-3546$

Éditeur

Presses universitaires de Bordeaux

\section{Édition imprimée}

Date de publication : 1 décembre 2010

ISBN : 978-2-86781-743-4

ISSN : $1168-5549$

\section{Référence électronique}

Jean-Luc Vannier, "L'involontaire psychique du Projet Professionnel Personnalisé », Communication et organisation [En ligne], 38 | 2010, mis en ligne le 19 décembre 2012, consulté le 30 avril 2019. URL : http://journals.openedition.org/communicationorganisation/1491; DOI : 10.4000/ communicationorganisation. 1491 


\title{
L'involontaire psychique du Projet Professionnel Personnalisé
}

\author{
Jeon-LUc VANNIER ${ }^{01}$ \\ "Dans le même fleuve deux fois nous descendons et ne descendons pas " ${ }^{02}$
}

Depuis quelques années, le Ministère français de l'Enseignement supérieur et de la recherche a mis en place au sein des Instituts Universitaires de Technologie, un séminaire - l'auteur précisera les raisons pour lesquelles il préfère ce mot à celui de cours - intitulé PPP ou « Projet Professionnel Personnalisé ». L'idée partait d'un simple constat: chaque année en France, plusieurs milliers d'étudiants, toutes filières confondues, stoppaient net leurs études en cours de cycle. Ils hésitaient à reprendre un cursus pour se retrouver finalement sur le marché du travail sans diplôme. Motif apparent de leur abandon: le constat, après quelques mois d'expérimentation, que la formation suivie ne correspondait pas à ce qu'ils " aimaient » ou "désiraient » faire. Décalage entre le choix d'une orientation professionnelle et l'expression dans l'après-coup, d'un désir individuel dont l'insatisfaction conduit à la prise de conscience. Ratage révélateur du symptôme. Davantage orientés - en théorie - vers les formations professionnalisantes et les cycles courts, les IUT ont bénéficié de cette innovation. Son indéniable succès vaut aujourd'hui au PPP d'être intégré sous une forme à peine modifiée à la première année des licences traditionnelles. Plus récemment, la Ministre Valérie Pécresse a mis l'accent sur «les dispositifs de soutien et d'accompagnement» - enseignants référents, tutorat pédagogique - dans son projet de "Nouvelle Licence ». Notons qu'une réforme en cours instaure un dispositif de "deux heures d'accompagnement personnalisé hebdomadaire " pour chaque lycéen. Lorsqu'il est dispensé par un professionnel de la psyché, le Projet Professionnel Personnalisé comporte un «involontaire psychique »: si

01. Jean-Luc Vannier est Psychanalyste (Beyrouth, Nice et Villefranche-sur-Mer), Chargé de cours à l'Université de Nice Sophia-Antipolis et à l'Edhec. Membre du Cercle psychanalytique « Le Pinacle de Beyrouth-NiceParis » et du Comité de rédaction de la revue libanaise de psychanalyse "Ashtarout ». Auditeur de l'Institut des Hautes Etudes de la Défense Nationale, il anime une émission hebdomadaire de psychanalyse sur Radio Chalom Nitsan.

02. Héraclite (cité par Sénèque dans « Lettres à Lucilius », Coll. « Bouquins », Éditions Robert Laffont, 1998). 
le séminaire ne saurait se transformer en séances formelles de psychothérapie, la qualité d'analyste, la place occupée et le discours tenu par ce dernier suscitent des réactions qui n'en sont pas très éloignées. À l'image du concept de "praticable " (Nassif, 1977), le PPP crée les conditions favorables d'un échange avec l'enseignant sur le devenir-être l'étudiant: celui-ci s'interroge, explore et se remet en question. Il fait par surcroît affleurer des « demandes » dont la nature correspond le plus souvent aux critères de souffrance semblables à ceux pour lesquels ces spécialistes sont amenés à proposer leur écoute. Plus qu'un outil de médiation dont le terme est depuis fort longtemps galvaudé, le PPP prend l'allure d'une " aire » ou d'une " analyse transitionnelle " à la mesure des « groupes restreints » (Anzieu 1980, 2007): il aide à franchir une étape et offre au sujet la possibilité d'advenir.

\section{Orientation professionnelle et connaissance de soi}

La fiche ministérielle d'instruction du PPP contient une formule intéressante, signifiante à plus d'un titre: elle demande à l'enseignant d'aider l'étudiant à «trouver un métier et pas seulement un emploi». Une telle exigence au contenu explicitement « identitaire » et sur laquelle les étudiants sont invités à réfléchir, évoque une dimension psychologisante de ce séminaire. Celle-ci se confirme en toutes lettres, ailleurs dans cette notice pédagogique: le PPP doit combiner "l'orientation professionnelle et la connaissance de soi ». Démarche d'autant plus ambitieuse que le marché et les conditions du travail pullulent désormais de références psychiques: le séquençage du travail, illustré par le nombre croissant en Europe des emplois précaires - travail à durée déterminée, tâches polyvalentes et mobilité géographique - a sérieusement altéré la correspondance traditionnelle, structurante, entre un capital de connaissances professionnelles et une identité personnelle. L'être humain "n'est » plus en mesure de répondre à la question posée sur la nature de son travail: « je suis». Il occupe parmi d'autres un "poste de travail » qui lui concède non sans un certain dédain, sa valeur laborieuse (Alain Touraine, 1969, 1973). Illustré par la tentative gouvernementale de mettre en place un « indice du bonheur » susceptible de pondérer humainement le PIB, le retour des affects accompagne par ailleurs les modalités d'appréhension du mal-être ou du stress au travail. Parallèlement à la rémunération, mesurable et comptable, croît la doléance ambiguë d'une "reconnaissance » individualisée des salariés et des cadres pour laquelle les entreprises privilégient souvent la formation à l'augmentation de salaire: contrairement à la matérialité de la seconde, la première propose l'acquisition d'un savoir supplémentaire qui flatte la confiance et renforce l'estime de soi. Le prix à payer se nomme «insertion professionnelle » avec toutes les images de contorsions, physiques comme psychiques, que ce terme charrie avec lui. Démarche ambitieuse, mais aussi risquée. Dans un monde où l'hyperconsommation développe le dogme d'un objet à bien vivre au-delà de son acquisition pour ses seules qualités fonctionnelles (Lipovetsky 2006), 
le PPP ne doit pas se faire subrepticement le complice d'une double illusion: celle d'une construction artificielle et d'une autonomie possible d'un « Moi » obsédé par l'adaptation à son environnement. « Moi » consumériste et performant, forgé de toutes pièces à partir d'un modèle qui prétendrait s'affranchir des individualités et auquel s'imposerait une "injonction à la jouissance " au lieu de cheminer sur la voie du désir (Vannier 2003).

\section{Il y a du transfert dans l'air}

En charge du PPP à aménager dans certaines des formations où j’interviens à l'IUT de Nice (TC, GEA, CS), j’invite toujours d'emblée mes étudiants, après la lecture d'un texte court qui fait référence au voyage et à la découverte d'autres cultures ainsi qu'aux bouleversements émotionnels et aux remises en question qu'ils suscitent, à réfléchir aux processus de l'altérité, point de passage obligé vers l'identité. Une altérité susceptible de commencer dans le séminaire: du voisin à l'autre en soi. Une introduction inhabituelle destinée à préciser un fait: le PPP se veut un "séminaire » et non un cours. Contrairement au Droit, à l'Économie, aux règles commerciales ou aux stratégies de marketing, le PPP ne contient pas un savoir unilatéral à transmettre. À moins de considérer l'existence d'un "savoir insu » dont les étudiants comprennent rapidement qu'ils en sont eux-mêmes les dépositaires. Comme certains le disent avec le sens frappant de la formule, "nous devenons l'objet du cours!". Premier contact avec une dimension inconsciente probablement renforcée par l'annonce de la qualité de psychanalyste de l'enseignant. Avec des conséquences immédiates pour des étudiants dont l'entrée à l'IUT colle au besoin affiché d'un "encadrement ", voire d'une "prise en charge ». Notions dont les cliniciens du divan savoureront toute la portée. Dès la première heure, il y a en effet du « transfert » dans l'air (Freud, 1895) et les ressources en puissance que recèle la "psychologie des profondeurs " ne laissent pas les étudiants indifférents. Les divers exercices réalisés progressivement au cours de l'année («La journée idéale de travail », « la Check List ») permettent pratiquement à chaque étudiant de s'exprimer « oralement » devant ses pairs, lesquels, n'en doutons pas, font preuve d'une oreille particulièrement attentive, étonnamment "empathique " aux propos de leurs camarades. Il est vrai qu'à cette période de post-adolescence, la recherche de nouvelles identifications, la volonté d'adhésion et d'appartenance au groupe, notamment au travers de l'explosion des réseaux sociaux, trahissent la nécessité de dire, tout comme celle d'être entendu. Qu'il s'agisse de commentaires critiques d'un CV ou d'une lettre de motivation, tous deux présentés sous la forme d'un transparent de façon à permettre la visualisation du document par l'ensemble du groupe, que l'étudiant soit chargé d'analyser et de résumer un article de presse dont le contenu croise toujours des problématiques professionnelle et personnelle (le code vestimentaire, la mise au placard dans l'entreprise, les ressources humaines, le stress, les relations intimes au travail, être mère et femme au travail, 
le marketing expérientiel, le suicide dans l'entreprise...), qu'interviennent enfin, et dans certains cas, des jeux de rôle (présentation devant un recruteur, annonce délicate d'un licenciement, négociation d'un congé de maternité, conflit hiérarchique au sein d'une entreprise...), les dynamiques de groupe élaborées à cette occasion montrent une finesse réactive de perception des étudiants. Ces derniers n'hésitent pas à mentionner à leurs camarades «l'ambivalence de leur discours ", le fossé existant entre leurs finalités déclarées et ce qu'ils ressentent des moyens mis en œuvre, parfois jugés inadéquats, pour les atteindre. Le patient, expliquait Lacan, dit toujours la vérité mais ne dit pas toute la vérité. À présentateur peu convaincu, public circonspect.

\section{Vignettes cliniques : "j'écoute même du Mozart!"}

Les choix en apparence "volontaires » se confrontent aux désirs inconscients. Principe de plaisir contre principe de réalité (Freud, 1911). Proposons à ce stade quelques vignettes. Ainsi cet étudiant d'une vingtaine d'années qui se destine à une carrière commerciale. Plutôt par défaut. Sa vraie passion, celle qui anime son regard comme celle qui accélère le débit de ses paroles lorsqu'il l'évoque, deux phénomènes immédiatement relevés par la classe, c'est le "foot » pratiqué pendant 17 ans sans interruption. "C'est toute ma vie » ajoute-t-il au cours de son intervention. Ses autres camarades l'interpellent: "pourquoi tu n'as pas continué? ". "Impossible » leur assène-t-il, avant de reconnaître qu'il n'a "jamais vraiment tenté de démarches en ce sens». Questionné, le terrain des incapacités physiques cède promptement la place aux interdits d'ordre psychique. Et chacun de se demander en silence ce que peut bien dissimuler cet «impossible », gouffre du Réel pour plagier les Lacaniens. Il en va de même pour cet autre étudiant qui, à la fin du séminaire de PPP, annonce à la classe qu'il va quitter l'IUT afin de reprendre des études de médecine. Études qu'il « a toujours eu envie de suivre». Prise de conscience tardive visant à dépasser une injonction parentale énigmatique et formulée depuis son plus jeune âge: «tu n'y arriveras pas». Quelques minutes de débat au sein de l'assistance suffisent pour balayer l'explication purement intellectuelle et laisser « librement associer » sur les non-dits familiaux. Honte, dissimulée sous la verbalisation de l'incompétence, de ne pouvoir financer des études longues, angoisse parentale devant un cheminement social susceptible d'éloigner leur progéniture de leur milieu habituel, projection inconsciente de leurs propres limites sur celles accordées aux ambitions de leur fils, aucune hypothèse n'est écartée si l'on veut bien en laisser la liberté aux participants. L'enfant compensateur pour le meilleur comme pour le pire (Israël, 1989). Au cours d'une présentation de $\mathrm{CV}$, un étudiant d'origine marocaine interrogé par ses camarades sur la raison qui l'avait conduit à ne pas mentionner sa pratique dialectale, doublée d'une connaissance de l'arabe classique, répondra avec une certaine candeur: « vous savez, j'écoute même du Mozart! ». Une bonne dizaine de minutes de "perlaboration " collective sur les processus d'acculturation suivra cette instant 
d'une exceptionnelle intensité. Un dernier exemple clinique illustrera mon propos: en début d'année, un étudiant affirme sur un ton péremptoire que l'argent constitue le motif essentiel de son choix pour une carrière commerciale. Un fort sentiment contre-transférentiel me convainc du contraire mais je laisse évidemment la liberté à l'étudiant d'exposer ses motivations. Lors de son passage trois semaines plus tard et sans aucune intervention particulière de ma part, ce dernier ne tiendra pas plus d'une minute: devant ses camarades ébahis, il «avouera » s'être trompé et surtout avoir cherché à "les tromper». Il y a bien le motif de l'argent mais plus par impérieuse nécessité que par préférence: malgré ses compétences, il veut suivre une formation courte pour s'établir et vivre sans être au crochet de ses parents. Tout le monde pense à une situation de survie économique. À la fin du cours, l'étudiant infirmera cette hypothèse: la situation financière aisée de ses parents s'éclipse devant l'existence d'un grave désordre familial qui rend intolérable pour lui le fait de continuer à vivre dans cet environnement. À la fin de l'année, il quittera l'IUT pour reprendre des "études longues en économie ». Anecdote supplétive intéressante: une étudiante qui effectue le même travail un peu après ne «cessera de mettre en avant" le thème des "relations et des soins avec les enfants". Ce que ne manqueront pas de lui faire remarquer ses camarades de classe qui relèvent le peu de liens avec une filière commerciale stricto sensu. Elle aussi abandonnera le cursus en fin de première année: il s'agissait de la «chérie » de l'étudiant précédent qui avait intégré le même groupe que son partenaire affectif. Elle poursuit à ce jour des études de psychologie spécialisées dans la petite enfance. Qu'on se rassure: le PPP ne triomphe pas seulement avec les ruptures de cursus! On peut inscrire à son crédit les poursuites d'études. Nombres d'étudiants qui pensaient "péniblement » achever les deux années de DUT se découvrent d'authentiques intérêts, voire des passions qui les incitent à prolonger brillamment leur parcours universitaire. Preuve que la période moratoire de l'adolescence, celle des expérimentations et des tâtonnements avant l'engagement (Gutton, 1996, 2000), s'étend bien au-delà des limites fixées par la médecine ou la psychiatrie.

\section{Frustration du réel}

Un autre exercice consiste à tenter de représenter le passage du choix imaginaire d'une profession, ponctué du "j’ai toujours rềvé de... » et guidé par le sentiment de toute puissance inhérent à la pensée magique de l'enfance, à la sélection réelle d'un métier contrainte par le principe de réalité à l'âge adulte. Le "super héros", "l'astronaute ", "l'espion " pour les garçons, "la chanteuse adulée de music hall » ou la " princesse " pour la petite fille laissent advenir, dans le schéma requis, des «chefs d'entreprises ", des "Business Women » ou des « experts en communication ». Le déplacement inconscient des ambitions infantiles vers le pouvoir de l'économie ou celui de la publicité n'a pas encore révélé tous ses avatars! Bordée de réflexions sur les codes psycho-sociaux qui préexistent 
à leur naissance et sont susceptibles d'influencer leur parcours ultérieur (Héritier, 1996, 2002), la réalisation de cet exercice s'accompagne, parallèlement à la reconnaissance et à l'intégration d'une limitation drastique du champ des possibles, d'un net sentiment de « frustration » et de « déception ». Freud n'aurait pas démenti. Tout en rappelant les rites de passages (Van Gennep, 1909), du premier jour de maternelle aux examens universitaires de fin d'année, ce travail met souvent à jour le poids de la «dette psychique » chez des étudiants qui admettent devoir supporter les effets verbalisés d'un "sacrifice " parental consenti pour leurs études au nom de "l'amour» (Freud, 1909). Finalement, autant de «séances » qui ne disent pas leur nom et à même de confirmer, d'infirmer, de modifier ou d'affiner des orientations professionnelles tout en contribuant à chaque fois à l'affirmation de personnalités.

\section{Rire du psychanalyste}

Signalons, en outre, des effets inattendus et collatéraux du «transfert ». Lors des devoirs semestriels sur table, plus d'un tiers des copies, cent quatrevingt au total pour les premières années, comportent des éléments souvent très personnels mais toujours éclairants sur les raisons d'un futur choix de métier. Avec cette question: "Quelle serait, selon vous, la journée de travail idéale? Expliquez en quoi elle vous ressemble ». La notion "d'idéale " réintroduit volontairement la dimension imaginative sans la cliver définitivement de la réalité. Une petite discussion sur la manière de construire une phrase avec la formule «l'idéal serait que... » permet ainsi d'indiquer que l'emploi du conditionnel n'implique ni ne rejette le but à atteindre. Parfois à la limite du sujet, la nature et le contenu des assertions frisent la confidence, pour ne pas dire la confession. Après un devoir sur table consacré au « mal-être dans le travail ", une jeune fille me joint le lendemain au téléphone. En pleurs, elle explique "avoir longuement éroqué dans sa copie le suicide de son père l'année passée et n'avoir pas compris les raisons d'une telle digression susceptible de la mettre hors sujet». Paroles, signifiants des mots "hors sujet» et abréaction sur un travail de deuil non accompli. "Le week-end qui a suivi votre cours", explique une autre étudiante dans sa copie, "j'ai longuement parlé avec mon père, actuellement sous antidépresseurs, qui a fini par m'avouer quil ne supportait plus le stress d'être manager». Les étudiants ont en quelque sorte inconsciemment profité des « capacités supposées » et spécifiques du correcteur - le "Sujet Supposé Savoir » de Lacan »- pour exprimer une interrogation, voire une souffrance directement liée à leur entrée dans le monde universitaire, marchepied de celui des adultes (Vannier, 2009) et à leur arrivée progressive dans celui du travail. Le mode transférentiel est d'ailleurs remarquable pour sa fulgurance: le fait de se présenter comme analyste au début du séminaire suscite de multiples réactions: interjections, sourires entendus, éclats intempestifs de rire, curiosité ou silence méfiant. Autant de mécanismes de défense qui recourent à la transformation d'un affect pénible en plaisir (Freud 1905, 1927). Ces derniers 
alternent avec des demandes plus assumées d'explications sur les différences avec les psychiatres et les psychologues. Jamais la mention qui intervient comme une sorte d'élément déclencheur ne laisse indifférent. Le transfert se manifeste le plus souvent par un scénario récurrent: il n'est pas rare qu'un étudiant vienne en fin de cours, affirme son "vif intérêt pour la psychologie » avant de se mettre soudainement à raconter ses souffrances personnelles. Formulation, sans trop le savoir, d'une demande exprimée sur eux-mêmes ou sur une personne qui leur est proche. Le séminaire de PPP offre l'assurance d'un cadre avec la régularité des interventions et la place occupée par le professeur. Malgré son caractère collectif, les éléments d'un " espace analytique » (Viderman, 1970) se mettent en place. Puisqu'il est question de place, il convient en outre de relever l'attachement de certains étudiants à celles qu'ils occupent, fonction souvent d'un regard direct ou latéral susceptible d'être porté sur l'intervenant. Un étudiant en Alternance qui se précipitait pour occuper une chaise située juste à l'angle de la classe, à cheval sur deux tables agencées en coin, s'étonnera de pouvoir expliquer son geste en fin d'année par les tragiques particularités de sa situation familiale: l'absence de parents tout deux décédés le mettait dans "l'inconfort» de s'installer délibérément sur l'une ou l'autre des rangées de la salle.

\section{Désarroi des enseignants}

Plus surprenant, le séminaire de PPP suscite également des questionnements personnels chez les enseignants. En témoignent les conclusions de deux réunions, à cinq années d'intervalle, avec des professeurs en charge du PPP dans la plupart des IUT de l'Hexagone. À Nantes en 2005, aucun des participants n'était, de près comme de loin, lié à la psychologie. Tous se félicitaient pourtant de "l'introduction de cette matière " au sein des IUT qu'ils considéraient comme une véritable "révolution" aux prolongements très positifs. Le PPP répondait, selon eux, à une "demande des étudiants", certes «pas toujours explicite de leur part » mais suffisamment ressentie par la communauté professorale pour qu'il y soit donné suite. Mais les enseignants, c'est là le point intéressant, ont généralement exprimé un certain "désarroi » devant la composante psychologique du PPP en relevant le fait qu'ils se sentaient "désarmés » pour affronter cette forme d'empathie: "orientation professionnelle, on sait faire ", déclarait l'un d'entre eux, "écouter la souffrance de l'étudiant qui vient en fin de cours se confier, on évite ». Ne les blâmons pas, chacun son métier. D'autres se sont carrément plaints d'une forme de "marginalisation", voire "d'étanchéité" au sein de leur IUT de la part des autres collègues. Rendons au passage justice à l'IUT de Nice où l'auteur de ces lignes n'a jamais rencontré ce type d'obstacles. Cinq années plus tard, le PPP fait figure de "valeur ajoutée » aux Instituts et devient susceptible de constituer dans les années à venir une de leurs plus précieuses références en termes d'images tant à l'extérieur de l'Université que vis-à-vis des étudiants. Dans 
le prolongement d'une réunion tenue à Paris en janvier 2011, il est désormais question de créer une liste de « référents PPP » désignés par les directeurs des IUT dans le but de renforcer les échanges entre les praticiens de la matière et d'en promouvoir les bienfaits auprès des différents responsables de l'Université. Si elle donne une sérieuse impulsion, cette perspective pose en retour plusieurs questions. Celle, en premier lieu, des précisions sur les contours et la nature des contenus à enseigner, tout en se méfiant clairement d'un souci national d'uniformisation. Celle, ensuite, de la capacité des enseignants à se frotter à l'inconscient de leurs étudiants. Et au leur propre.

\section{Emotions et agir}

En témoignent dans un autre registre les polémiques qui ont accompagné la rentrée scolaire 2010 et ont clairement posé le problème, à leur demande, de l'encadrement psychologique des enseignants des collèges et des lycées. Les quelque 850000 enseignants qui se sont épanchés dans la presse sur les conditions -psychologiques- d'exercice de leur métier le montrent: aucun d'entre eux ne remet en cause son acquis intellectuel. À raison, la profession se sent généralement bien armée pour chacune des matières à enseigner. C'est ailleurs que le bât blesse: angoissés face à leurs nouvelles responsabilités, ces enseignants reconnaissent mezza voce leur impréparation psychique. Mais pourquoi cette anxiété alors que leurs connaissances dans les disciplines, dûment sanctionnées par un diplôme et une formation, devraient logiquement les tenir à l'écart d'une telle crainte? Ce qui se joue dans ces plaintes ne concerne pas le cognitif mais l'affectif. L'assurance du savoir à transmettre ne garantit pas le fait de transmettre le savoir. Elle ne met pas les professeurs à l'abri d'autres défis: celles de leur propre "savoir insu " susceptible, s'ils n'ont pas suffisamment réglé cette dimension de leur histoire individuelle et familiale, de les rendre vulnérables lorsqu'ils se trouvent confrontés aux émotions et autres motions pulsionnelles désirantes - agressivité verbale, physique ou comportement ouvertement séducteur qui sont les deux faces d'une même souffrance - de leurs élèves. L'adolescent - et les étudiants des Universités ne sont pas encore pour la plupart d'entre eux, sortis de cette catégorie - plongent non sans tourment dans l'agir: actes qui reflètent leur lutte intérieure contre les effets du cataclysme pubertaire nourri du surgissement de la pulsion sexuelle. Un de mes amis, professeur de mathématique en Lycée, a été un jour interpellé par une jeune fille de quatorze ans qui avait remarqué des traces de craie sur sa joue: "voulez-vous que je vous lèche? " lui a-t-elle lancé crûment devant les autres élèves! Les conseillers d'orientations - ultime exemple de ma démonstration - ne remplissent pas non plus leur rôle: à entendre les étudiants, particulièrement véhéments à ce sujet, ces conseillers se réfugient le plus souvent derrière les feuilles de notations ou les résultats d'un test psychologique pour formuler des orientations évasives. Rappelons à ce titre qu'un test qui ne serait pas suivi d'un entretien personnalisé et individuel 
ne possède aucune valeur. La remarque "ils ne nous connaissent pas " revient fréquemment dans les propos des élèves interrogés.

Finalement, le PPP recouvre un enjeu qui, tant pour les étudiants que pour les enseignants, dépasse les fonctions officielles qui lui ont été attribuées. Les premiers l'ont, si l'on ose dire, définitivement adopté. En charge de cette matière, les seconds ont également compris son intérêt, sans toutefois résoudre la complexité des divers volets de son exploitation. Preuve, s'il en est, des difficultés à manier la dimension psychique dans une communauté humaine. Charité bien ordonnée commençant par soi-même, l'auteur de ces lignes reconnaîtra qu'il en aura probablement autant appris que ses étudiants sur le Projet Professionnel Personnalisé.

\section{BIBLIOGRAPHIE}

ANZIEU, Didier, MARTIN, Jacques-Yves, « La dynamique des groupes restreints », Coll. « Quadrige », Paris, PUF, 2007.

FREUD, Sigmund, «Études sur l'hystérie », Paris, PUF, 1956, rééd. 1975

FREUD, Sigmund, «Formulations sur les deux principes du cours des événements psychiques », OC, vol. XI, Paris, PUF, 1998.

FREUD, Sigmund, « Remarques sur un cas de névrose obsessionnelle, L'homme aux rats », in « Cinq psychanalyses », Paris, PUF, 1954, rééd. 1984.

FREUD, Sigmund, « Le mot d'esprit et sa relation à l'inconscient »,Paris, Gallimard, 1988.

FREUD, Sigmund, « L'humour », OC, vol. XVIII, Paris, PUF, 1994.

GUTTON, Philippe, «Adolescens », Paris, PUF, Coll. Le fil rouge, 1996.

GUTTON, Philippe, "Psychothérapie et adolescence », Paris, PUF, Coll. Le fil rouge, 2000.

HERITIER, Françoise, «Masculin/Féminin. La pensée de la différence », Paris, Odile Jacob, 1996.

HERITIER, Françoise, « Masculin/Féminin II », Paris, Odile Jacob, 2002.

ISRAEL, Lucien, « Boiter n'est pas pécher », Paris, Denoël 1989, rééd. 2010 Eres.

LIPOVETSKY, Gilles, «Le bonheur paradoxal, Essai sur la société d'hyperconsommation », Gallimard, 2006.

NASSIF, Jacques, «Freud, l'Inconscient, Sur les commencements de la psychanalyse », Éditions Galilée, 1977.

TOURAINE, Alain, « La société postindustrielle », Denoël, 1969

VAN GENNEP, Arnold, « Les rites de passage », Éditions A. et J. Picard, 1981.

VANNIER, Jean-Luc, «L'injonction à la jouissance ou la rue Monnot revisitée », in «Subjectivité et appartenances, Dynamiques inconscientes des cultures », Le Coq Héron, $\mathrm{N}^{\circ} 175$, Erès, décembre 2003. 
VANNIER, Jean-Luc, «Le stress pubertaire ou les maux de l'adolescence, Eléments pour une approche comparative franco-libanaise », 11 septembre 2002, site: psychanalyse in situ: www.psychanalyse-in-situ.fr/boite_a/vannier.html

VANNIER, Jean-Luc, «SOS Étudiants PACA », site: Nice-Premium, 13 juillet 2009, http://www.nice-premium.com/politique, 3/1-edito-du-psy-sos-etudiants-paca,4811.html

VIDERMAN, Serge, « La construction d'un espace analytique », Paris, Denoël, 1970

Résumé : L'instauration du PPP répond à un double souci d'orientation professionnelle et d'accompagnement personnalisé de l'étudiant. Pour peu qu'il soit mis en œuvre par un spécialiste de la psyché avec l'assentiment bienveillant de l'équipe enseignante, ce second volet ouvre sur une série de phénomènes psychiques et d'expériences transférentielles dignes d'intérêt pour la communauté pédagogique. Il révèle surtout cette dimension sous-jacente et inconsciente de l'encadrement, voire celle de la «prise en charge » et de ce « savoir insu », autant d'éléments recherchés par les étudiants qui s’inscrivent dans les IUT. Cet aspect apparaît amplement au point de devenir lui-même objet du séminaire et sujet de débats dans les différents exercices, dynamiques de groupe, travaux de présentations et exposés oraux accomplis au cours de l'année. On ne peut d'ailleurs que constater une «demande », le plus souvent explicite, des étudiants sur ce fragment d'eux-mêmes auquel ils semblent attacher une grande importance pour leur réussite sur le marché de l'emploi. En conséquence et à titre de témoignage, l'auteur se propose de communiquer les résultats de son travail en la matière dans les différents départements de l'IUT de Nice Sophia Antipolis où il enseigne depuis plusieurs années (Techniques de Commercialisation, Gestion des Administrations et des Entreprises, Carrières Sociales).

Mots-clés : Psychanalyse, IUT, Université, Enseignants, Projet professionnel personnalisé, Étudiants, Savoir, Transfert, orientation, Psyché, Métier, Souffrance, Réalité, Enfance, Séminaire, Affects, Inconscient.

\begin{abstract}
The introduction of PPP serves the student in two ways: 1 . with vocational guidance and 2. with personalized support. When the program is implemented by a specialist of psychology along with the support of the faculty, this second part opens a wide range of psychological phenomena and transferential experiences that would be of interest to the educational community as a whole. It especially reveals the underlying and unconscious dimension of this structure and the dynamic involved in it, even that of the desire for support including the "unknown knowledge"; all of which is sought after by the students enrolled in IUT. Eventually this aspect in itself grows to the point of becoming the topic of the seminars and the subject of debates in various exercises, group dynamics, work and oral presentations made during the academic year. We will also see often explicit "application" of this fragment of themselves from the students to which they give great importance both on their way and in the job market. Resultantly and as testimony the author proposes to present the results of his work in the different departments (TC, GEA and CS) where he has been teaching for several years.

Key words: Psychoanalysis, University, Professors, Personalized and professional project, Students, Knowledge, Transfert, Subconscious, Psychological request, Jobs, Suffering, Childhood, Seminar, Emotions, Orientation, Reality.
\end{abstract}

